\title{
Ácido acetilsalicílico na prevenção da pré-eclâmpsia: uma revisão baseada na evidência
}

Sara Santos Ferreira, ${ }^{1-2}$ Ana Carolina Martins, ${ }^{1,3}$ Ana Cláudia Magalhães, ${ }^{1,4}$ Hélder Martins $^{5-6}$

\section{RESUMO}

Objetivo: Rever a evidência atual sobre o efeito do ácido acetilsalicílico (AAS) na prevenção da pré-eclâmpsia. Fontes de dados: MEDLINE e sítios de medicina baseada na evidência.

Métodos de revisão: Pesquisa de normas de orientação clínica (NOC), ensaios clínicos aleatorizados e controlados (ECAC), revisões sistemáticas (RS) e meta-análises (MA), publicados entre 13/11/2005 e 13/11/2015, utilizando os termos MESH 'preeclampsia' e 'aspirin' e os termos DeCS 'pre-eclâmpsia' e 'aspirina'. Para atribuição do nível de evidência (NE) e força de recomendação (FR) foi utilizada a Strenght of Recommendation Taxonomy, da American Academy of Family Physicians.

Resultados: Foram selecionados 14 de entre 296 artigos encontrados: sete MA, três RS, três NOC e um ECAC. Globalmente, as RS e MA demonstram o benefício do AAS na prevenção da pré-eclâmpsia, principalmente em mulheres de alto risco. Apenas uma RS com NE 2 não encontrou evidência para suportar o uso desta profilaxia nas mulheres em risco de pré-eclâmpsia. Os estudos que analisam separadamente as mulheres de baixo risco na população-alvo verificaram uma ausência de benefício do AAS neste grupo de mulheres. As NOC recomendam o uso de AAS na prevenção da pré-eclâmpsia em mulheres de alto risco, desaconselhando o seu uso nas mulheres de baixo risco. O ECAC faz a mesma recomendação relativamente às mulheres de alto risco.

Conclusões: A evidência disponível indica que o AAS em baixa dose tem benefício quando usado como medicação preventiva nas mulheres em risco de pré-eclâmpsia, com evidência clara do seu benefício nas mulheres de alto risco (FR A), sendo desaconselhado o seu uso em mulheres de baixo risco (FR A). Existe ainda necessidade de mais estudos de elevada qualidade, de metodologia homogénea e amostras relevantes que suportem esta evidência.

Palavras-chave: Pre-eclâmpsia; Aspirina.

\section{INTRODUÇÃO}

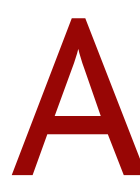
pré-eclâmpsia é uma das patologias obstétricas mais graves, sendo uma das principais causas de morbimortalidade materna e fetal. ${ }^{1}$ Habitualmente manifesta-se na segunda metade da gravidez, complicando 2 a $8 \%$ das gestações ${ }^{2} \mathrm{e}$ contribuindo para mais de 100.000 mortes maternas por ano a nível global. ${ }^{3}$ Segundo a International Socie-

1. Médica Interna de Medicina Geral e Familiar

2. USF Infesta, Unidade Local de Saúde de Matosinhos

3. USF Fiães, ACeS Feira Arouca

4. UCSP Moimenta da Beira, ACeS Douro Sul

5. Médico Especialista em Medicina Geral e Familiar

6. USF Maresia, Unidade Local de Saúde de Matosinhos ty for the Study of Hypertension in Pregnancy (ISSHP), a pré-eclâmpsia é definida pelo início de hipertensão após as 20 semanas de gestação, combinada com proteinúria (>300mg/dia) ou outras disfunções de órgão-alvo, como disfunção renal ou hepática, complicações neurológicas ou hematológicas, disfunção útero-placentária ou restrição de crescimento intrauterino (RCIU). ${ }^{4}$ A pré-eclâmpsia está ainda associada a parto pré-termo, quer espontâneo quer iatrogénico, e a nascimento de recém-nascidos pequenos para a idade gestacional (RN-PIG). ${ }^{5}$ Adicionalmente, as crianças nascidas de mães com pré-eclâmpsia têm maior risco de displasia broncopulmonar e paralisia cerebral associadas 
ao parto pré-termo. ${ }^{5}$ De um modo geral, a pré-eclâmpsia diminui a qualidade de vida relacionada com a saúde e aumenta o risco de depressão pós-parto. ${ }^{5}$

Apesar da causa da pré-eclâmpsia permanecer desconhecida, parece estar relacionada com alterações ao nível da formação e organização da placenta, com consequentes defeitos na perfusão útero-placentária e indução de um estado de hipercoagulabilidade. ${ }^{6}$ Este último aspeto parece estar relacionado com uma deficiência relativa da produção de prostaciclina (um vasodilatador) e produção excessiva de tromboxano (uma prostaglandina vasoconstritora sintetizada durante a agregação plaquetária). ${ }^{1}$ Tem sido proposto que a terapêutica com ácido acetilsalicílico (AAS) em baixa dose inibe a produção de tromboxano, reduzindo a vasoconstrição e a hipercoagulabilidade da placenta, ${ }^{7}$ hipótese que motivou a realização de vários ensaios clínicos a avaliar o uso de agentes antiplaquetários, como o AAS na prevenção da pré-eclâmpsia. ${ }^{1}$ Adicionalmente tem sido sugerido que a eficácia do AAS pode estar relacionada com a idade gestacional em que é iniciada a terapêutica. ${ }^{8}$ A normal formação e estruturação da placenta compreende a invasão das artérias espirais do endométrio pelas células do trofoblasto, processo que ocorre a partir da oitava semana de gestação, acreditando-se estar completa entre as 16 e as 20 semanas de gestação. Pelo exposto, alguns autores defendem haver maior benefício na introdução do AAS numa fase precoce da gravidez. ${ }^{9}$

O médico de família, sendo a base do sistema de saúde, é o primeiro profissional a providenciar cuidados obstétricos à população, tendo um papel importante no rastreio e abordagem inicial das mulheres com risco ou diagnóstico de pré-eclâmpsia. Neste sentido, os autores pretendem rever a evidência mais recente sobre a eficácia do AAS na prevenção da pré-eclâmpsia.

\section{MÉTODOS}

Foi realizada uma pesquisa de normas de orientação clínica (NOC), ensaios clínicos aleatorizados e controlados (ECAC), revisões sistemáticas (RS) e meta-análises (MA), publicados entre 13 de novembro de $2005 \mathrm{e}$ 13 de novembro de 2015, nas línguas portuguesa e inglesa, indexados nas bases de dados da National Guideline Clearinghouse, National Institute for Health and Care Excellence (NICE) Guidelines Finder, Canadian Me- dical Association Practice Guidelines Infobase, The Cochrane Library, Database of Abstracts of Reviews of Effectiveness (DARE), Bandolier, Evidence Based Medicine Online e MEDLINE, utilizando os termos MESH 'preeclampsia'e 'aspirin' e os termos DeCS 'pre-eclâmpsia' e 'aspirina'.

Os critérios utilizados para a inclusão dos artigos consistiram em: população-alvo constituída por mulheres grávidas sem pré-eclâmpsia na gravidez atual; intervenção terapêutica com AAS (sem restrições de dosagem; isolado ou em associação com outros agentes antiagregantes plaquetários); em comparação com placebo ou nenhum tratamento e outcome relacionado com a ocorrência de pré-eclâmpsia.

Foram utilizados como critérios de exclusão: estudos não-randomizados, artigos duplicados, artigos de opinião, artigos de revisão clássica de tema, sumários de sítios na Internet e artigos discordantes do objetivo da revisão. Foram também excluídos ensaios clínicos incluídos em RS ou MA selecionadas.

Para estratificar o nível de evidência (NE) dos estudos e a força de recomendação (FR) foi utilizada a Strenght of Recommendation Taxonomy (SORT), ${ }^{7}$ da American Academy of Family Physicians.

A seleção dos artigos para revisão foi feita em duplicado pelos dois primeiros autores que, perante dúvidas, discutiram em conjunto a inclusão/exclusão do artigo com uma taxa de concordância final de $100 \%$. A leitura integral foi realizada e a avaliação da qualidade e NE dos artigos incluídos discutida por todos os autores.

\section{RESULTADOS}

Da pesquisa efetuada resultou um total de 296 artigos, dos quais 14 cumpriam todos os critérios de inclusão: sete MA, três RS, três NOC e um ECAC. A Figura 1 representa o fluxograma de seleção dos estudos. Os Quadros I, II e III resumem as características dos estudos selecionados para revisão.

\section{Mulheres de alto risco versus mulheres de baixo risco}

Dos estudos incluídos, três $\mathrm{MA}^{10-12}$ avaliaram o efeito do AAS na prevenção da pré-eclâmpsia, estratificando as mulheres de acordo com o seu grau de risco. Para tal selecionaram ECAC cuja intervenção consistia no uso de AAS $v$ s placebo em mulheres grávidas divididas em dois subgrupos: alto e baixo risco para pré-eclâmp- 


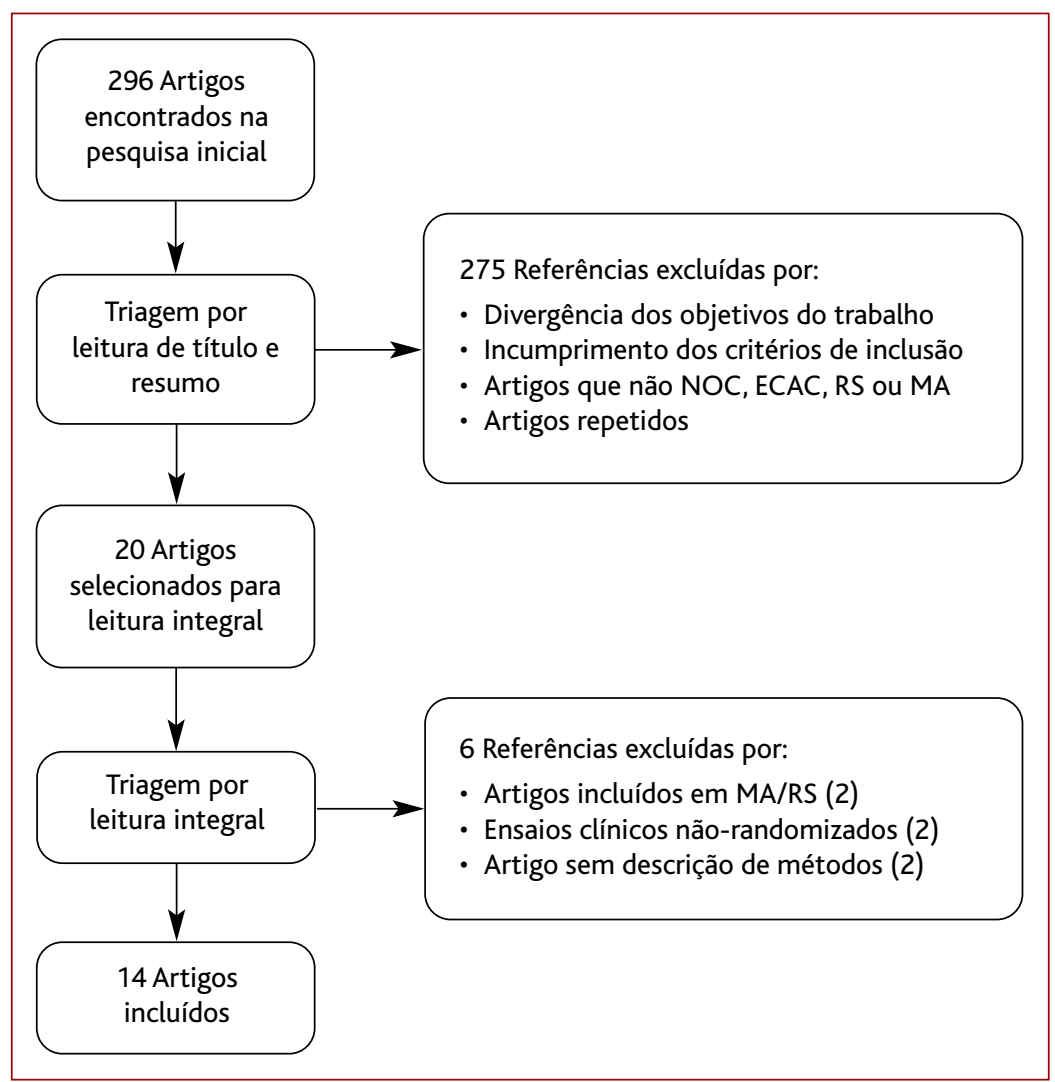

Figura 1. Fluxograma de seleção dos artigos.

Legenda: ECAC - Ensaios clínicos aleatorizados e controlados; MA - Metanálise; NOC - Normas de orientação clínica; RS - Revisão sistemática. cia e qualidade dos estudos incluídos, pelo que foi atribuído a este trabalho NE 2. Rossi e colaboradores ${ }^{10}$ chegam a uma conclusão diferente. Os autores não conseguem encontrar diferenças significativas entre o grupo tratado com AAS e o tratado com placebo, quer para as mulheres de alto risco $(\mathrm{OR}=0,72$; IC95\%, $0,51-1,00 ; p=0,05)$ quer para as de baixo risco $(\mathrm{OR}=0,82$; IC95\%, 0,65-1,04; $p=0,10$ ), concluindo não haver evidência que suporte a administração de AAS em baixa dose para a prevenção da pré-eclâmpsia. Contudo, o artigo não especifica as dosagens de AAS utilizadas nos diferentes estudos, nem aquilo que os autores consideram como baixa dose de AAS. Por este motivo e por não especificar com clareza os critérios de seleção dos estudos incluídos foi atribuído a este trabalho NE 2.

Henderson e colaboradores ${ }^{13}$ conduziram uma RS com o objetivo de avaliar o efeito do AAS na prevenção da pré-eclâmpsia apenas em mulheres de alto risco. Para tal selecionaram ECAC cuja intervenção consistia na toma de AAS em baixa dose comparado com placebo ou nenhum tratamento na população supracitada. Após análise, os autores concluem haver benefício no uso de AAS em baixa dose para prevenção da pré-eclâmpsia em mulheres de alto risco (RR=0,76 com IC95\%, 0,620,95). À semelhança de Henderson e colaboradores, ${ }^{13}$ também Ayala e colaboradores ${ }^{14}$ procuraram avaliar o efeito do AAS na prevenção da pré-eclâmpsia em mulheres de alto risco, verificando uma redução ligeira na incidência de pré-eclâmpsia com a intervenção $(p=0,04$ com número necessário tratar $[\mathrm{NNT}]=16)$. Ambos os trabalhos cumprem critérios de boa qualidade, pelo que lhes foi atribuído NE 1.

Askie e colaboradores, ${ }^{15}$ numa MA publicada na Lancet, e Duley e colaboradores, ${ }^{16}$ numa RS da Cochrane, tiveram como objetivo avaliar o efeito, não só do AAS, mas também de outros agentes antiagregantes plaquetários (dipiridamol, heparina e ozagrel) na prevenção da pré-eclâmpsia. Ambos os estudos incluíram mu- 


\section{QUADRO I. Metanálises}

\begin{tabular}{|c|c|c|c|c|}
\hline Referência & População & Intervenção & Resultados & NE \\
\hline $\begin{array}{l}\text { Ruano e } \\
\text { colaboradores, } \\
2005^{11}\end{array}$ & $\begin{array}{l}\text { Mulheres grávidas, } \\
\text { divididas em } 2 \text { grupos: } \\
\text { alto risco e baixo risco } \\
\text { de pré-eclâmpsia. } \\
22 \text { ECAC } \\
\text { (grupo de alto risco - } \\
17 \text { ECAC; grupo de baixo } \\
\text { risco - } 5 \text { ECAC) } \\
n=33.598 \\
\text { ( } 16.898 \text { mulheres no } \\
\text { grupo de alto risco e } \\
16.700 \text { no grupo de } \\
\text { baixo risco) }\end{array}$ & $\begin{array}{l}\text { AAS ( } 50 \text { a } 150 \mathrm{mg} / \mathrm{dia}) \\
\text { vs placebo }\end{array}$ & $\begin{array}{l}\text { Grupo de alto risco: } \\
\text { » Pequena redução no risco de pré-eclâmpsia } \\
\text { (RR=0,87; IC95\%, 0,79-0,96). } \\
\text { Grupo de baixo risco: } \\
\text { » O tratamento com AAS em baixa dose não } \\
\text { reduz de forma significativa o risco de } \\
\text { pré-eclâmpsia (RR=0,95; IC95\%, 0,81-1,11) } \\
\text { Globalmente: } \\
\text { » Não há correlação entre a dosagem de AAS e a } \\
\text { prevenção de pré-eclâmpsia (coeficiente de } \\
\text { correlação } r=0,064 \text { ) } \\
\text { » Não há correlação óbvia entre idade gestacional } \\
\text { de início do AAS e a prevenção da pré-eclâmpsia }\end{array}$ & 1 \\
\hline $\begin{array}{l}\text { Askie e } \\
\text { colaboradores, } \\
2007^{15}\end{array}$ & $\begin{array}{l}\text { Mulheres grávidas com } \\
\text { risco de pré-eclâmpsia, } \\
\text { independentemente do } \\
\text { grau de risco. } \\
31 \text { ECAC } \\
n=32.217\end{array}$ & $\begin{array}{l}\text { AAS isolado ( } 50 \text { a } \\
150 \mathrm{mg} / \text { dia) vs placebo } \\
\text { ou nenhum tratamento } \\
\text { ( } 27 \mathrm{ECAC} ; n=31.678 \text { ) } \\
\text { AAS combinado com } \\
\text { dipiridamol vs placebo } \\
\text { ou nenhum tratamento } \\
(n=177) \\
\text { Dipiridamol e/ou } \\
\text { heparina ou ozagrel } \\
\text { isolado vs placebo ou } \\
\text { nenhum tratamento } \\
\text { ( } n=362 \text { ) }\end{array}$ & $\begin{array}{l}\text { » O uso de agentes antiplaquetários foi associado } \\
\text { a uma redução moderada }(10 \%) \text {, mas consistente } \\
\text { no risco de pré-eclâmpsia }(\mathrm{RR}=0,90 ; \mathrm{IC} 95 \% \text {, } \\
0,84-0,97 ; \mathrm{NNT}=114) \\
\text { » Não há evidência clara de que o uso de agentes } \\
\text { antiplaquetários tenha maior ou menor benefício } \\
\text { na redução do risco relativo num subgrupo } \\
\text { particular de doentes }\end{array}$ & 1 \\
\hline $\begin{array}{l}\text { Bujold e } \\
\text { colaboradores, } \\
2010^{9}\end{array}$ & $\begin{array}{l}\text { Mulheres grávidas, com } \\
\text { risco de pré-eclâmpsia } \\
\text { independentemente do } \\
\text { grau de risco. } \\
34 \text { ECAC } \\
n=11.348\end{array}$ & $\begin{array}{l}\text { AAS ( } 50 \text { a } 150 \mathrm{mg} / \mathrm{dia}) \\
\text { isolado ou combinado } \\
\text { com dipiridamol } \\
\text { ( } 300 \mathrm{mg} / \mathrm{dia} \text { ) vs placebo } \\
\text { ou nenhum tratamento } \\
\text { NOTA: } \\
\text { As variáveis foram } \\
\text { controladas para a } \\
\text { idade gestacional de } \\
\text { início da intervenção }\end{array}$ & $\begin{array}{l}\text { Grupo que iniciou a intervenção até às } 16 \\
\text { semanas de gestação inclusive: } \\
\text { » Redução significativa da incidência de } \\
\text { pré-eclâmpsia no grupo de mulheres que iniciou a } \\
\text { intervenção até às } 16 \text { semanas de gestação } \\
\text { inclusive (RR=0,47; IC95\%, 0,34-0,65) } \\
\text { Grupo que iniciou a intervenção após as } 16 \\
\text { semanas de gestação: } \\
\text { " Não houve diferenças na incidência de } \\
\text { pré-eclâmpsia no subgrupo de mulheres que } \\
\text { iniciou a intervenção após as } 16 \text { semanas de } \\
\text { gestação (RR=0,81; IC95\%, } 0,63-1,03)\end{array}$ & 1 \\
\hline
\end{tabular}




\begin{tabular}{|c|c|c|c|c|}
\hline Referência & População & Intervenção & Resultados & NE \\
\hline Trivedi, $2011^{12}$ & $\begin{array}{l}\text { Mulheres grávidas, } \\
\text { divididas em } 2 \text { grupos: } \\
\text { alto risco e baixo risco } \\
\text { de pré-eclâmpsia. } \\
19 \text { ECAC } \\
\text { (grupo de alto risco - } \\
14 \text { ECAC; grupo de baixo } \\
\text { risco - } 5 \text { ECAC) } \\
n=28.237 \\
\text { (11.687 mulheres no } \\
\text { grupo de alto risco e } \\
16.550 \text { no grupo de } \\
\text { baixo risco) }\end{array}$ & $\begin{array}{l}\text { AAS (50 a } 150 \mathrm{mg} / \mathrm{dia}) \\
\text { vs placebo }\end{array}$ & $\begin{array}{l}\text { Grupo de alto risco: } \\
\text { » Redução de } 21 \% \text { no risco de pré-eclâmpsia } \\
\text { (RR=0,79; IC95\%, 0,65-0,97; NNT=19) } \\
\text { Grupo de baixo risco: } \\
\text { » O tratamento com AAS não reduz de forma } \\
\text { significativa o risco de pré-eclâmpsia (RR=0,86; } \\
\text { IC95\%, 0,64-1,1; NNT=200) } \\
\text { Globalmente: } \\
\text { » Não há correlação entre a dosagem de AAS e a } \\
\text { prevenção de pré-eclâmpsia (coeficiente de } \\
\text { correlação } r=0,13 ; \text { IC } 95 \%, 0,34-0,55 ; p=0,58 \text { ) }\end{array}$ & 2 \\
\hline $\begin{array}{l}\text { Rossi e } \\
\text { colaboradores, } \\
2011^{10}\end{array}$ & $\begin{array}{l}\text { Mulheres grávidas, } \\
\text { divididas em } 2 \text { grupos: } \\
\text { alto risco e baixo risco } \\
\text { de pré-eclâmpsia. } \\
10 \text { ECAC } \\
\text { (grupo de alto risco - } 7 \\
\text { ECAC; grupo de baixo } \\
\text { risco - } 3 \text { ECAC) } \\
n=20.630 \\
\text { (10.729 mulheres no } \\
\text { grupo de alto risco e } \\
9.901 \text { no grupo de } \\
\text { baixo risco) }\end{array}$ & $\begin{array}{l}\text { AAS em baixa dose vs } \\
\text { placebo } \\
\text { NOTA: } \\
\text { O artigo não especifica } \\
\text { as doses de AAS } \\
\text { utilizadas nos diferentes } \\
\text { estudos }\end{array}$ & $\begin{array}{l}\text { Grupo de alto risco: } \\
\text { »A incidência de pré-eclâmpsia foi similar entre } \\
\text { os } 2 \text { grupos (OR=0,72; IC } 95 \%, 0,51-1,00 ; p=0,05) \\
\text { Grupo de baixo risco: } \\
\text { » Não houve diferenças na incidência de } \\
\text { pré-eclâmpsia (OR=0,82; IC } 95 \%, 0,65-1,04 ; \\
p=0,10)\end{array}$ & 2 \\
\hline $\begin{array}{l}\text { Roberge e } \\
\text { Giguère e } \\
\text { colaboradores, } \\
2012^{17}\end{array}$ & $\begin{array}{l}\text { Mulheres grávidas com } \\
\text { alto risco de } \\
\text { pré-eclâmpsia. } \\
4 \text { ECAC } \\
n=392\end{array}$ & $\begin{array}{l}\text { AAS ( } 50 \text { a } 150 \mathrm{mg} / \mathrm{dia}) \\
\text { isolado ou combinado } \\
\text { com dipiridamol } \\
\text { ( } \leq 300 \mathrm{mg} / \mathrm{dia} \text { ) vs } \\
\text { placebo ou nenhum } \\
\text { tratamento }\end{array}$ & $\begin{array}{l}\text { „ O AAS iniciado até às } 16 \text { semanas de gestação } \\
\text { inclusive reduz o risco global de pré-eclâmpsia } \\
\text { (RR=0,52; IC95\%, 0,38-0,76; } p<0,01) \text { e do risco } \\
\text { de pré-eclâmpsia grave ( } R R=0,22 ; \mathrm{IC} 95 \% \text {, } \\
\text { 0,08-0,57; heterogeneidade } 0 \%) \\
\text { "Não houve diferenças no risco de pré-eclâmpsia } \\
\text { moderada (RR=0,81; IC95\%, 0,33-1,96) }\end{array}$ & 2 \\
\hline
\end{tabular}

lheres grávidas em risco de pré-eclâmpsia, independentemente do grau de risco, e verificaram uma redução moderada, mas significativa, do risco de pré-eclâmpsia $\left(10 \%{ }^{15}\right.$ e $17 \%,{ }^{16}$ respetivamente) com o uso de agentes antiagregantes plaquetários. No entanto, a
RS da Cochrane refere ainda que a magnitude da redução foi superior nas mulheres de alto risco (25\%; $\mathrm{NNT}=19)$ face às de moderado risco $\left(14 \%\right.$; NNT=119). ${ }^{16}$ Já a MA publicada na Lancet refere não haver evidência clara de que a intervenção tenha maior ou menor 


\begin{tabular}{|c|c|c|c|c|}
\hline Referência & População & Intervenção & Resultados & NE \\
\hline $\begin{array}{l}\text { Roberge e Villa } \\
\text { e colaboradores, } \\
2012^{28}\end{array}$ & $\begin{array}{l}\text { Mulheres grávidas com } \\
\text { risco de pré-eclâmpsia, } \\
\text { independentemente do } \\
\text { grau de risco. } \\
5 \text { ECAC } \\
n=556\end{array}$ & $\begin{array}{l}\text { AAS ( } 50 \text { a } 150 \mathrm{mg} / \text { dia) } \\
\text { isolado ou combinado } \\
\text { com dipiridamol } \\
\text { (<300mg/dia) vs } \\
\text { placebo ou nenhum } \\
\text { tratamento } \\
\text { NOTA: } \\
\text { A intervenção teve } \\
\text { início } \leq 16 \text { semanas de } \\
\text { gestação }\end{array}$ & $\begin{array}{l}\text { „O AAS em baixa dose, iniciado até às } 16 \text { semanas } \\
\text { de gestação inclusive, esteve associado a uma } \\
\text { redução de } 89 \% \text { no risco de pré-eclâmpsia } \\
\text { pré-termo (RR=0,11; IC95\%, 0,04-0,33; } p<0,01 \text {; } \\
\text { heterogeneidade } 0 \%) \\
\text { »Não houve diferenças no risco de pré-eclâmpsia } \\
\text { de termo (RR=0,98; IC95\%, 0,42-2,33; } p=0,97 \text {; } \\
\text { heterogeneidade } 54 \%)\end{array}$ & 1 \\
\hline
\end{tabular}

Legenda: AAS - ácido acetilsalicílico; ECAC - ensaio clínico aleatorizado e controlado; IC95\% - intervalo de confiança a 95\%; NE - nível de evidência; NNT - número necessário tratar; OR - Odds Ratio; RR - risco relativo.

benefício na redução do risco relativo num subgrupo particular de doentes (NNT global=114). ${ }^{15}$ Apesar de a análise avaliar a ação de vários agentes antiagregantes plaquetários na prevenção da pré-eclâmpsia, Duley e colaboradores ${ }^{16}$ salientam que a maior parte da evidência (51 de 59 ECAC) se refere ao AAS em baixa dose, pelo que deve ser este o agente de escolha. Por sua vez, Askie e colaboradores ${ }^{15}$ não especificam nenhum agente antiagregante plaquetário em particular, embora o AAS também tenha sido o agente mais utilizado na intervenção (27 de 31 ECAC). Ambos os trabalhos cumprem critérios de boa qualidade dos ECAC selecionados, pelo que foi atribuído a ambos NE 1 .

Roberge e Giguère e colaboradore ${ }^{17}$ conduziram uma MA com o objetivo de avaliar o benefício do AAS em baixa dose, iniciado até às 16 semanas de gestação, na prevenção de pré-eclâmpsia grave e de pré-eclâmpsia moderada em mulheres de alto risco para pré-eclâmpsia. Para tal selecionaram ECAC cuja intervenção consistia no uso de AAS em baixa dose, isoladamente ou em associação com dipiridamol, iniciado até à idade gestacional supracitada, controlado com placebo ou nenhum tratamento. Os autores concluem que o início de AAS em baixa dose até às 16 semanas de gestação, inclusive, se associa a uma diminuição do risco global de pré-eclâmpsia em mulheres de alto risco $(\mathrm{RR}=0,52 ; \mathrm{IC} 95 \%, 0,38-0,76 ; p<0,01)$. Analisando separadamente os resultados para o risco de pré-eclâmpsia grave e moderada, os autores verificaram que essa redução de risco é sobretudo para a pré-eclâmpsia grave, em que a redução do risco relativo se aproxima dos $90 \%$, não havendo diferenças para a pré-eclâmpsia moderada (RR=0,81; IC95\%, 0,33-1,96). O facto de esta RS ter incluído um pequeno número de estudos com um número reduzido de participantes pode ter conduzido a vieses significativos, pelo que foi atribuído a este trabalho NE 2.

A United States Preventive Services Task Force (USPSTF) publicou, em 2014, uma NOC relativa ao uso de AAS em baixa dose na prevenção da morbimortalidade por pré-eclâmpsia. ${ }^{18}$ Esta entidade concluiu com moderada certeza haver evidência substancial do benefício do AAS em baixa dose na redução do risco de pré-eclâmpsia em mulheres de alto risco. Neste sentido, recomenda o uso de AAS em baixa dose em mulheres grávidas de alto risco para pré-eclâmpsia (FR B).

A ISSHP emitiu, em 2014, uma NOC para a abordagem das doenças hipertensivas na gravidez, de entre as quais se salienta a pré-eclâmpsia. ${ }^{19}$ Esta entidade conclui haver boa evidência para recomendar o uso de AAS em baixa dose para prevenção da pré-eclâmpsia em mulheres de alto risco (FR A), não havendo evidência suficiente para fazer esta recomendação em mulheres de baixo risco (FR A).

$\mathrm{O}$ National Institute for Health and Care Excellence (NICE) publicou, em 2011, uma NOC sobre a aborda- 


\begin{tabular}{|c|c|c|c|c|}
\hline Referência & População & Intervenção & Resultados & NE \\
\hline $\begin{array}{l}\text { Duley e } \\
\text { colaboradores, } \\
2007^{16} \\
\text { (Cochrane) }\end{array}$ & $\begin{array}{l}\text { Mulheres grávidas } \\
\text { em risco de } \\
\text { pré-eclâmpsia, } \\
\text { classificadas nos } \\
\text { seguintes } \\
\text { subgrupos: alto, } \\
\text { moderado e } \\
\text { moderado-baixo } \\
\text { risco para } \\
\text { pré-eclâmpsia } \\
59 \text { ECAC } \\
n=37.560\end{array}$ & 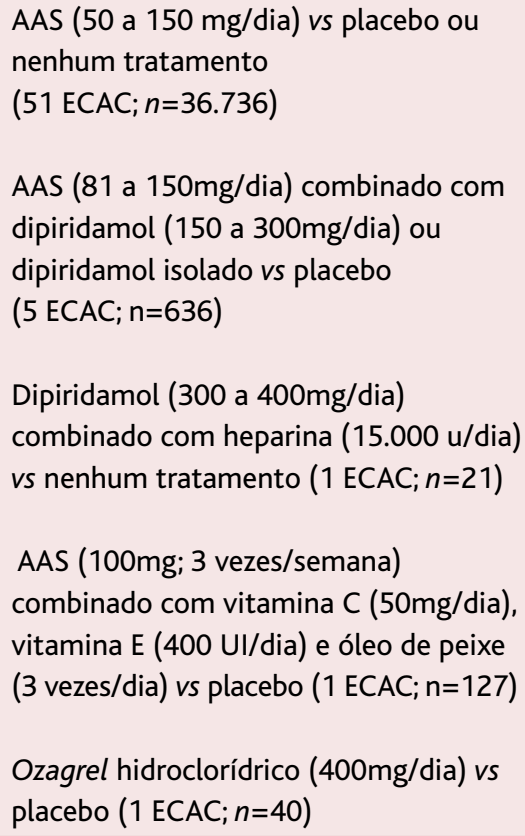 & 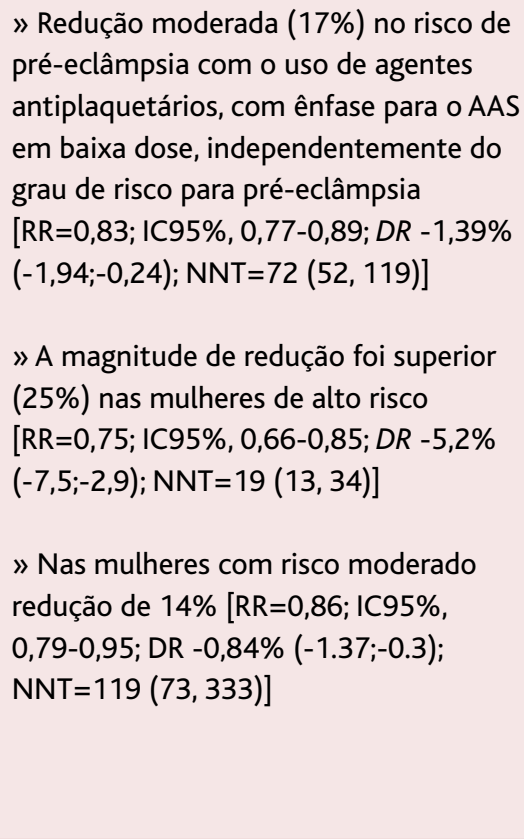 & 1 \\
\hline $\begin{array}{l}\text { Henderson e } \\
\text { colaboradores, } \\
2014^{13}\end{array}$ & $\begin{array}{l}\text { Mulheres grávidas } \\
\text { com alto risco de } \\
\text { pré-eclâmpsia } \\
13 \text { ECAC } \\
n=24.474\end{array}$ & $\begin{array}{l}\text { AAS ( } 50 \text { a } 150 \mathrm{mg} / \text { dia) vs placebo ou } \\
\text { nenhum tratamento } \\
\text { NOTA: } \\
\text { A intervenção foi iniciada entre as } 12 \text { e } \\
\text { as } 16 \text { semanas de gestação inclusive }\end{array}$ & $\begin{array}{l}\text { » Redução de } 24 \% \text { no risco de } \\
\text { pré-eclâmpsia (RR=0,76; IC } 95 \% \text {, } \\
0,62-0,95)\end{array}$ & 1 \\
\hline
\end{tabular}

Legenda: AAS - ácido acetilsalicílico; DR - diferença de risco; ECAC - ensaio clínico aleatorizado e controlado; IC95\% - intervalo de confiança a 95\%; $\mathrm{NE}$ - nível de evidência; NNT - número necessário tratar; RR - risco relativo.

gem das doenças hipertensivas na gravidez, elaborada pelo Royal College of Obstetricians and Gynaecologists em colaboração com o National Collaborating Centre for Women's and Children's Health. ${ }^{20}$ Estas entidades concluem existir evidência suficiente para recomendar AAS em baixa dose na prevenção da pré-eclâmpsia, sendo que o risco-benefício do seu uso é dependente do risco de desenvolver pré-eclâmpsia. Assim, existe uma clara indicação em aconselhar AAS em baixa dose para profilaxia da pré-eclâmpsia em mulheres de alto risco (FR A), contrariamente ao que se verifica para as mulheres de baixo risco (FR A). Embora admitam que os estudos não são consensuais relativamente aos critérios para classificação de mulheres de moderado risco, estas entidades recomendam também o uso de AAS em baixa dose na presença de pelo menos dois fatores de risco moderado para a doença (FR B). Os fatores considerados pela NICE para estratificação das mulheres em alto e moderado risco para pré-eclâmpsia encontram-se listados na Tabela II.

Gillon e colaboradores ${ }^{21}$ realizaram uma RS das recomendações de NOC internacionais sobre a abordagem de doenças hipertensivas na gravidez, onde se inclui a pré-eclâmpsia. Os autores recomendam o uso de AAS em baixa dose para a prevenção da pré-eclâmpsia em mulheres de alto risco (FR A); o mesmo não é reco- 


\section{QUADRO III. Ensaio clínico}

\begin{tabular}{|c|c|c|c|c|}
\hline Referência & População & Intervenção & Resultados & NE \\
\hline $\begin{array}{l}\text { Ayala e } \\
\text { colaboradores, } \\
2013^{14}\end{array}$ & $\begin{array}{l}\text { Mulheres grávidas com idade } \\
\geq 18 \text { anos, de nacionalidade } \\
\text { espanhola, com um risco de } \\
\text { hipertensão gestacional ou } \\
\text { pré-eclâmpsia superior à } \\
\text { generalidade da população } \\
\text { obstétrica, vigiadas num hospital } \\
\text { obstétrico (unidade de alto risco) } \\
\mathrm{n}=350\end{array}$ & $\begin{array}{l}\text { AAS (100mg/dia) vs } \\
\text { placebo } \\
\text { NOTA: } \\
\text { Os participantes foram } \\
\text { randomizados em seis grupos de } \\
\text { acordo com o tratamento (AAS } \\
\text { ou placebo) e a hora de } \\
\text { administração: ao acordar; } 8 \mathrm{~h} \\
\text { depois de acordar; e ao deitar. A } \\
\text { intervenção teve início entre as } \\
12 \text { e as } 16 \text { semanas de gestação, } \\
\text { inclusive }\end{array}$ & $\begin{array}{l}\text { AAS vs Placebo: } \\
\text { » Redução ligeira da incidência } \\
\text { de pré-eclâmpsia ( } p=0,041 ; \\
\text { NNT=16) } \\
\text { AAS ao deitar ou } 8 \text { h após } \\
\text { acordar vs AAS de manhã ou } \\
\text { placebo: } \\
\text { » Redução marcada da incidência } \\
\text { de pré-eclâmpsia ( } p<0,001 ; \\
\text { NNT=9) }\end{array}$ & 1 \\
\hline
\end{tabular}

Legenda: AAS - ácido acetilsalicílico; NE - nível de evidência; NNT - número necessário tratar.

mendado em mulheres de baixo risco (FR A).

Uma limitação importante, referida pelos autores da maior parte dos estudos incluídos nesta revisão, reside na disparidade de critérios para definir mulheres grávidas de alto e de baixo risco para pré-eclâmpsia, o que leva os autores a sugerir a realização de mais estudos para perceber concretamente quais os fatores que conferem maior risco para esta doença. ${ }^{11,13,16,20}$ Alguns trabalhos definem com clareza quais os fatores de risco que motivaram a inclusão dos diferentes estudos, definindo como mulheres de alto risco aquelas com, pelo menos, um fator de risco para pré-eclâmpsia e mulheres de baixo risco aquelas que não apresentam nenhum desses fatores. ${ }^{11-12}$ A NICE, na sua NOC de $2011,{ }^{20}$ e a Cochrane, na sua RS de 2007, ${ }^{16}$ fazem uma divisão diferente dos fatores de risco, considerando fatores de alto risco e fatores de risco moderado para pré-eclâmpsia. As Tabelas I e II apresentam todos os fatores de risco para pré-eclâmpsia considerados nos estudos incluídos nesta revisão.

\section{Dose de AAS}

Ruano e colaboradores ${ }^{11}$ e Trivedi ${ }^{12}$ analisaram, nas suas MA, a correlação entre as dosagens de AAS utilizadas nos diferentes estudos incluídos e a prevenção da pré-eclâmpsia, não havendo correlação em nenhum dos trabalhos. Os autores não conseguem, assim, tirar conclusões acerca da melhor dose de AAS para prevenção da pré-eclâmpsia, recomendando de forma genérica AAS em baixa-dose, sem especificar a dose. Da mesma forma, Henderson e colaboradores ${ }^{13}$ não foram capazes de demonstrar qual a dose ótima de AAS a administrar. Por sua vez, o ECAC de Ayala e colaboradores ${ }^{14}$ utilizou na sua intervenção $100 \mathrm{mg}$ de AAS por dia, concluindo que esta será a dose mínima recomendada para a prevenção da pré-eclâmpsia em mulheres de alto risco.

A USPSTF ${ }^{18}$ e a ISSHP ${ }^{19}$ são consensuais em recomendar o uso de AAS em baixa dose para a prevenção da pré-eclâmpsia em mulheres de alto risco. A USPSTF ${ }^{18}$ conclui com moderada certeza haver evidência substancial para recomendar o uso de AAS na dose de 60 a $150 \mathrm{mg} /$ dia (FR B), enquanto a ISSHP ${ }^{19}$ conclui haver razoável evidência para recomendar o uso de AAS na dose de 75 a 162mg/dia (FR B). Gillon e colaboradores ${ }^{21}$ na sua RS das recomendações de NOC internacionais recomendam o uso de AAS na dose de 60 a 162mg/dia (FR A). Por sua vez, a NICE $^{20}$ considera que a dose mínima recomendada de AAS deverá ser $75 \mathrm{mg} /$ dia, tendo em conta o perfil de segurança demonstrado do AAS nesta dose e a magnitude de redução do risco de pré-eclâmpsia (FR A). Por apurar fica o eventual benefício de doses mais elevadas de AAS em mulheres de alto risco. 


\begin{tabular}{|c|c|c|}
\hline Referência & País & Recomendações \\
\hline $\begin{array}{l}\text { National } \\
\text { Institute for } \\
\text { Health and Care } \\
\text { Excellence, } \\
2011^{20}\end{array}$ & $\begin{array}{l}\text { Reino } \\
\text { Unido }\end{array}$ & $\begin{array}{l}\text { Aconselhar as mulheres grávidas com alto risco de pré-eclâmpsia a iniciar } 75 \mathrm{mg} / \text { dia de AAS desde } \\
\text { as } 12 \text { semanas de gestação até ao parto (FR A) } \\
\text { Aconselhar mulheres com mais de um fator de risco moderado para pré-eclâmpsia a iniciar AAS } \\
\text { ( } 75 \mathrm{mg} / \text { dia) desde as } 12 \text { semanas de gestação até ao parto (FR B) } \\
\text { O AAS em baixa dose como forma de prevenção da pré-eclâmpsia não é recomendado para } \\
\text { mulheres com baixo risco de pré-eclâmpsia (FR A) }\end{array}$ \\
\hline $\begin{array}{l}\text { United States } \\
\text { Preventive } \\
\text { Services Task } \\
\text { Force, } 2014^{18}\end{array}$ & EUA & $\begin{array}{l}\text { O AAS em baixa dose ( } 60 \text { a 150mg/dia) pode ser usado como medicação preventiva depois das } 12 \\
\text { semanas de gestação em mulheres de alto risco para pré-eclâmpsia (FR B) }\end{array}$ \\
\hline $\begin{array}{l}\text { International } \\
\text { Society for the } \\
\text { Study of } \\
\text { Hypertension in } \\
\text { Pregnancy, } 2014^{19}\end{array}$ & - & $\begin{array}{l}\text { O AAS em baixa dose está recomendado para a prevenção da pré-eclâmpsia em mulheres de alto } \\
\text { risco (FR A) } \\
\text { O AAS em baixa dose não está recomendado para a prevenção da pré-eclâmpsia em mulheres de } \\
\text { baixo risco (FR A) } \\
\text { O AAS deve ser: } \\
\text { - Dado em baixa dose ( } 75 \text { a } 162 \mathrm{mg} / \mathrm{dia}) \text { (FR B); } \\
\text { - Administrado à hora de deitar (FR B); } \\
\text { - Iniciado após o diagnóstico de gravidez, mas antes das } 16 \text { semanas de gestação (FR B); } \\
\text { - Continuado até ao parto (FR C). }\end{array}$ \\
\hline $\begin{array}{l}\text { Gillon e } \\
\text { colaboradores, } \\
2014^{21} \\
\text { (revisão } \\
\text { sistemática das } \\
\text { recomendações } \\
\text { de NOC } \\
\text { internacionais) }\end{array}$ & - & $\begin{array}{l}\text { O AAS em baixa dose não está recomendado para a prevenção da pré-eclâmpsia em mulheres de } \\
\text { baixo risco (FR A) } \\
\text { O AAS em baixa dose ( } 60 \text { a } 162 \mathrm{mg} / \text { dia) está recomendado para a prevenção da pré-eclâmpsia em } \\
\text { mulheres de alto risco (FR A) } \\
\text { O AAS está recomendado desde o início da gravidez e até ao parto (FR A) }\end{array}$ \\
\hline
\end{tabular}

Legenda: AAS - ácido acetilsalicílico; EUA - Estados Unidos da América; FR - força de recomendação; NE - nível de evidência; NOC - normas de orientação clínica.

\section{Idade gestacional de início do AAS}

Para além de analisar a correlação entre a dosagem de AAS e a prevenção da pré-eclâmpsia, Ruano e colaboradores ${ }^{11}$ procuram também analisar a correlação entre as idades gestacionais de início do tratamento com AAS e a prevenção da pré-eclâmpsia, não encontrando qualquer tipo de correlação. Os autores não conseguem, assim, concluir acerca da melhor altura para iniciar o tratamento com AAS. Da mesma forma, Henderson e colaboradores ${ }^{13}$ e Trivedi ${ }^{12}$ não conseguiram avaliar a influência da idade gestacional de início da intervenção no outcome final. Por sua vez, o ECAC de Ayala e colaboradores ${ }^{14}$ iniciou a sua intervenção até às 16 semanas de gestação inclusive, concluindo que o AAS deve ser iniciado no máximo até esta idade gestacional. Da mesma forma, Roberge e Giguère e colaboradores ${ }^{17}$ 
incluíram, na sua MA, ECAC cuja intervenção era iniciada até às 16 semanas de gestação, concluindo que o AAS deve ser iniciado até às 16 semanas de gestação, inclusive, para prevenção da pré-eclâmpsia em mulheres de alto risco.

Bujold e colaboradores ${ }^{9}$ realizaram uma MA com o objetivo de avaliar especificamente a influência da idade gestacional de início do AAS na incidência de pré-eclâmpsia. Para tal selecionaram ensaios clínicos prospetivos, controlados e randomizados, cuja intervenção consistia no uso de AAS em baixa dose, isoladamente ou em associação com dipiridamol, controlado com placebo ou nenhum tratamento, numa amostra de mulheres de alto risco para pré-eclâmpsia. Para atingir os objetivos propostos, os autores estratificaram os resultados de acordo com a idade gestacional de início da intervenção: até às 16 semanas inclusive ou após as 16 semanas de gestação. Os autores concluem haver uma redução significativa da incidência de pré-eclâmpsia quando o AAS é iniciado até às 16 semanas de gestação inclusive (RR=0,47; IC95\%, 0,34-0,65), não havendo diferenças na incidência de pré-eclâmpsia no subgrupo de mulheres que iniciou a intervenção após as 16 semanas de gestação (RR=0,81; IC95\%, 0,63-1,03). Ficou ainda por demonstrar se existe uma idade gestacional a partir da qual não existe benefício em iniciar AAS. Para diminuir vieses, os autores excluíram artigos quasi-randomizados, falhas na dupla ocultação e perdas de follow-up superiores a $20 \%$. Neste contexto foi atribuído ao estudo NE 1.

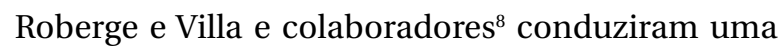
MA com o objetivo de avaliar o benefício do AAS em baixa dose, iniciado também até às 16 semanas de gestação, no risco de desenvolvimento de pré-eclâmpsia pré-termo e de pré-eclâmpsia de termo. Os autores definem pré-eclâmpsia pré-termo como aquela que se associa a parto até às 37 semanas completas de gestação e pré-eclâmpsia de termo aquela que se associa a parto após as 37 semanas de gestação. Para atingir os objetivos propostos, os autores incluíram ECAC cuja intervenção consistia no uso de AAS em baixa dose, com ou sem dipiridamol, controlado com placebo ou nenhum tratamento, em mulheres grávidas com risco de pré-eclâmpsia (independentemente do grau de risco) e com a idade gestacional supracitada. Os autores verificaram que o início de AAS em baixa dose até às 16

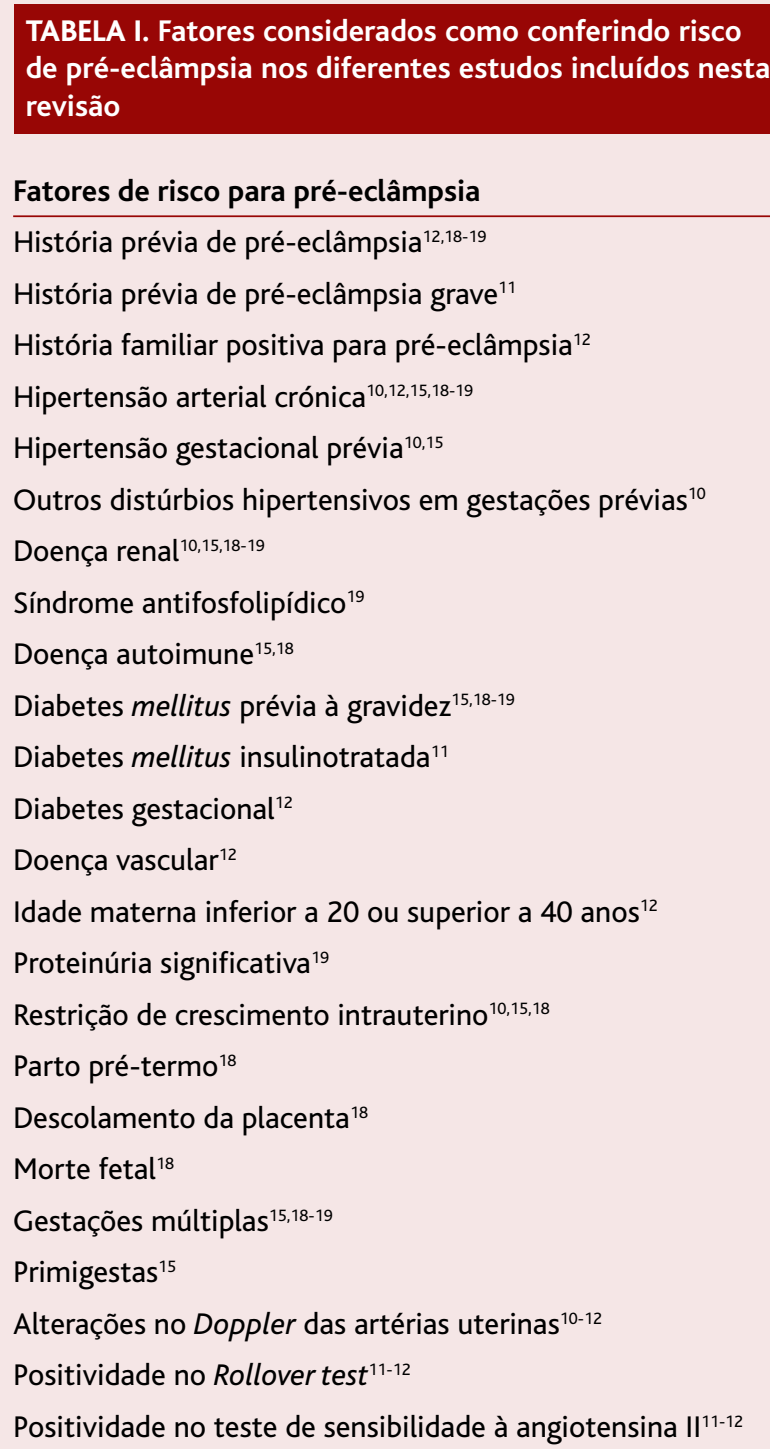

semanas de gestação, inclusive, resultou numa diminuição de $89 \%$ no risco de pré-eclâmpsia pré-termo ( $\mathrm{RR}=0,11$ com IC95\%, 0,04-0,33; $p<0,01$ ), não havendo diferenças no risco de pré-eclâmpsia de termo $(\mathrm{RR}=0,98$ com IC95\%, 0,42-2,33; $p=0,97$ ). Os autores justificam os resultados obtidos com a grande heterogeneidade (54\%) entre os estudos que avaliaram o risco de pré-eclâmpsia de termo, colocando ainda a hipótese de a terapêutica com AAS diminuir o risco de pré-eclâmpsia grave, ou seja, aquela que se associa a parto pré-termo, não reduzindo o risco de pré-eclâmpsia moderada, ou seja, aquela que permite partos de termo. Esta 


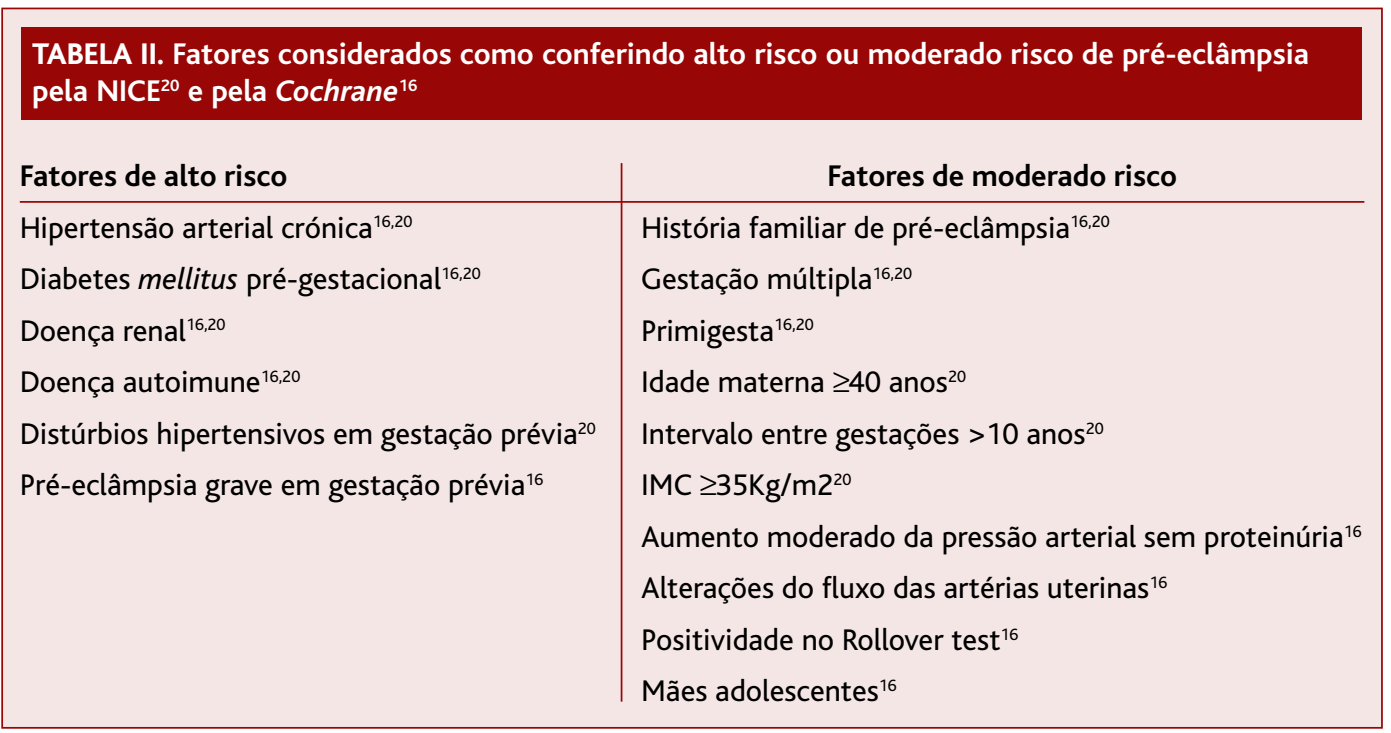

Legenda: IMC - índice de massa corporal; $\geq$ - maior ou igual.

MA cumpre critérios de boa qualidade, pelo que lhe foi atribuído NE 1.

A USPSTF ${ }^{18}$ conclui com moderada certeza haver evidência substancial para recomendar o início de AAS após as 12 semanas de gestação em mulheres grávidas de alto risco para pré-eclâmpsia (FR B), não se manifestando sobre a idade gestacional até à qual deve ser mantida a terapêutica com AAS. Da mesma forma, a $\mathrm{NICE}^{20}$ considera importante o início do AAS às 12 semanas de gestação em mulheres de alto risco para pré-eclâmpsia, referindo ter sido esta a idade gestacional mais precoce para a qual foi identificado benefício do uso de AAS (FR A). Relativamente à descontinuação do tratamento, a NICE ${ }^{20}$ refere não existir evidência de qual será a idade gestacional ótima. A ISSHP ${ }^{19}$ refere haver razoável evidência para recomendar que o AAS seja iniciado logo após o diagnóstico de gravidez e antes das 16 semanas de gestação (FR B) e evidência inconsistente para recomendar a manutenção da terapêutica com AAS até ao parto (FR C). Gillon e colaboradores, ${ }^{21}$ na sua RS das recomendações de NOC internacionais, recomendam o início do AAS logo no início da gravidez que deve ser mantido até ao parto (FR A).

\section{Hora de administração do AAS}

Para além de avaliar o efeito do AAS na prevenção da pré-eclâmpsia em mulheres de alto risco, Ayala e cola- boradores ${ }^{14}$ procuraram também perceber se esse efeito estaria dependente da hora de administração. Para tal conduziram um estudo prospetivo, randomizado e duplamente cego, tendo randomizado 350 mulheres em seis grupos, de acordo com o tratamento (AAS ou placebo) e a hora de administração (ao acordar, oito horas depois de acordar e ao deitar). Os resultados foram analisados para a comparação "AAS vs placebo" e, posteriormente, para a comparação "AAS ao deitar ou oito horas após acordar $v s$ AAS de manhã ou placebo". Quando comparado com o placebo, o AAS, independentemente da hora de administração, reduziu ligeiramente a incidência de pré-eclâmpsia $(\mathrm{NNT}=16)$. Este efeito é claramente ampliado quando a ingestão do AAS é feita à tarde ou ao deitar (NNT=9), levando os autores a recomendar a administração de AAS ao deitar, para prevenção da pré-eclâmpsia.

A ISSHP ${ }^{19}$ é a única NOC a emitir recomendações sobre a hora de administração do AAS, referindo haver evidência razoável para recomendar que a administração de AAS seja feita ao deitar (FR B).

\section{Perfil de segurança do AAS}

Uma das principais preocupações relacionadas com a terapêutica com AAS durante a gravidez é a possibilidade de um aumento de complicações hemorrágicas na grávida ou no recém-nascido. ${ }^{15}$ Estas preocupações 
são em parte dissipadas por alguns dos estudos incluídos nesta revisão.

A incidência de descolamento prematuro da placenta, de hemorragia anteparto e de hemorragia pós-parto com a terapêutica com AAS foram consistentemente avaliados em vários trabalhos. Rossi e colaboradores ${ }^{10}$ verificaram a ocorrência materna de descolamento da placenta e necessidade de transfusão sanguínea em dois estudos envolvendo mulheres de alto risco e em dois outros estudos envolvendo mulheres de baixo risco. Contudo, não encontraram diferenças entre as mulheres grávidas tratadas com AAS e as tratadas com placebo (AAS em baixa dose: 28/752 $(3,7 \%) v$ s placebo: $26 / 772$ (3,3\%); $Z=0,36 ; P=0,72 ; \mathrm{OR}=1,10 \mathrm{com}$ IC95\%, 0,60-1,90), indicando que o AAS em baixa dose é seguro (NE 2). Da mesma forma, Bujold e colaboradores ${ }^{9} \mathrm{e}$ Henderson e colaboradores ${ }^{13}$ não encontraram diferenças significativas na incidência de descolamento da placenta (RR=0,62 com IC95\%, $0,08-5,03^{9} \mathrm{e}$ $\mathrm{RR}=1,17$ com IC95\%, 0,93-1,48, ${ }^{13}$ respetivamente). Henderson e colaboradores ${ }^{13}$ ressalvam, contudo, que as baixas taxas de eventos reportados podem ter induzido vieses importantes na interpretação deste outcome. Por este motivo e para este outcome foi atribuído a este trabalho NE 2.

Em relação à incidência de hemorragia pós-parto, Henderson e colaboradores ${ }^{13}$ e Ayala e colaboradores ${ }^{14}$ não encontraram diferenças entre os grupos tratados com AAS e os tratados com placebo ( $R R=1,02 \mathrm{com}$ IC95\%, 0,96-1,09 ${ }^{13}$ e $p=0,303,{ }^{14}$ respetivamente) (NE 1 ), resultados corroborados por Trivedi ${ }^{12}$ na sua metanálise de 2011 (NE 2). Em relação à incidência de hemorragia anteparto, Ayala e colaboradores ${ }^{14}$ não encontraram diferenças significativas entre o AAS em baixa dose e o placebo $(p=0,415)$ (NE 1). Rossi e colaboradores ${ }^{10}$ não conseguiram avaliar a relação entre hemorragia anteparto e pós-parto e o uso de AAS para profilaxia da pré-eclâmpsia, pelas disparidades encontradas na definição de hemorragia pós-parto entre os estudos incluídos e pela elevada subjetividade na avaliação das perdas sanguíneas.

Askie e colaboradores ${ }^{15}$ e Duley e colaboradores ${ }^{16}$ avaliaram o efeito e a segurança não só do AAS, como também de outros agentes antiplaquetários na prevenção da pré-eclâmpsia. Nenhum dos trabalhos encontrou diferenças na incidência de descolamento pre- maturo da placenta (RR=1,13 com IC95\%, 0,87-1,48 ${ }^{15} \mathrm{e}$ $\mathrm{RR}=1,10$, IC95\%, 0,89-1,37, ${ }^{16}$ respetivamente). Paralelamente, Askie e colaboradores ${ }^{15}$ não encontraram diferenças na incidência de hemorragia anteparto $(\mathrm{RR}=1,02$, com IC95\%, 0,90-1,15) e na incidência de hemorragia pós-parto (RR=1,06 com IC95\%, 1,00-1,13). Os autores sublinham, no entanto, que as conclusões relativas à incidência de hemorragia pós-parto devem ser interpretadas com cautela, pelas dificuldades na mensuração e definição de hemorragia pós-parto nos diferentes estudos incluídos. Por este motivo e para estes outcomes foi atribuído a este trabalho NE 2.

A incidência de hemorragia neonatal e a possibilidade de ocorrência de anomalias do desenvolvimento são outras das preocupações associadas à exposição ao AAS durante a gestação. Relativamente ao primeiro outcome, Trivedi ${ }^{12}$ e Duley e colaboradores ${ }^{16}$ não encontraram diferenças significativas na incidência de hemorragia neonatal (NE 2). Em relação ao segundo outcome, Henderson e colaboradores ${ }^{13}$ e Duley e colaboradores ${ }^{16}$ não relataram potenciais efeitos prejudiciais ou diferenças no desenvolvimento até aos 18 meses de idade, salvaguardando que efeitos raros ou a longo prazo não foram identificados e que mais estudos são necessários (NE 2). Da mesma forma, Askie e colaboradores $^{15}$ não relatam aumento do risco de anomalias congénitas, nem problemas no desenvolvimento até cerca dos dois anos de idade (NE 2).

As NOC incluídas nesta revisão são consensuais em afirmar a segurança do AAS em baixa dose. ${ }^{18-20} \mathrm{~A}$ USPSTF $^{18}$ refere que esta terapêutica não está associada à ocorrência de descolamento prematuro da placenta, hemorragia pós-parto ou danos fetais, como hemorragia intracraniana e anomalias congénitas, enquanto a ISSHP ${ }^{19}$ refere que a terapêutica com AAS não aumenta nem diminui o risco de abortamento espontâneo, não havendo evidência de teratogenicidade ou outros efeitos adversos pediátricos a curto ou a longo prazo.

\section{CONCLUSÕES}

De acordo com a evidência atual disponível, pode concluir-se que o AAS em baixa dose tem benefício quando usado como medicação preventiva nas mulheres em risco de pré-eclâmpsia, com evidência clara do seu benefício nas mulheres de alto risco (FR A). Não 
existe, contudo, evidência suficiente para recomendar esta profilaxia nas mulheres de baixo risco (FR A). Estas conclusões são suportadas por um elevado número de estudos, a maioria de boa qualidade, com resultados consistentes e orientados para o paciente.

Globalmente, as RS e MA de boa qualidade incluídas nesta revisão demonstraram que o uso de AAS em baixa dose conduzia a reduções significativas do risco relativo de pré-eclâmpsia em mulheres de alto risco., ${ }^{911,13,16}$ Apenas uma RS com NE 2 não encontrou evidência suficiente para suportar o uso desta profilaxia nas mulheres em risco de pré-eclâmpsia, independentemente do seu grau de risco. ${ }^{10}$ Os estudos que analisaram separadamente as mulheres de baixo risco na populaçãoalvo verificaram uma ausência de benefício do AAS neste subgrupo de mulheres. ${ }^{10-12}$

Relativamente à dose de AAS a administrar, os estudos são consensuais em recomendar AAS em baixa dose (FR A). ${ }^{11-14}$ Contudo, a maior parte dos estudos inclui ECAC com disparidades importantes nas dosagens de AAS, pelo que à luz da evidência disponível não é possível determinar a dose ótima de AAS a ministrar para prevenção da pré-eclâmpsia.

No que respeita à idade gestacional de início da terapêutica profilática com AAS, não existe consenso entre os estudos, que apresentam critérios de inclusão divergentes relativamente a este parâmetro. Ruano e colaboradores ${ }^{11}$, Henderson e colaboradores ${ }^{13}$ e Trivedi $^{12}$ não conseguiram determinar a idade gestacional ótima para início da profilaxia com AAS. Não obstante, a MA de Bujold e colaboradores ${ }^{9}$ e o ECAC de Ayala e colaboradores,$^{14}$ dois estudos de boa qualidade com NE 1 , concluem haver maior benefício no início da terapêutica com AAS até às 16 semanas de gestação inclusive, sendo esta a idade gestacional recomendada para início da profilaxia (FR A).

O ECAC de Ayala e colaboradores ${ }^{14}$ foi o único estudo a avaliar objetivamente o efeito da cronoterapia com AAS na prevenção da pré-eclâmpsia. À luz deste estudo de boa qualidade (NE 1) é possível recomendar, com uma FR B, que a profilaxia com AAS seja administrada preferencialmente ao deitar.

Alguns estudos avaliaram ainda a incidência de efeitos adversos relacionados com a terapêutica, verificando que o uso de AAS em baixa dose não esteve associado a aumento da incidência de efeitos adversos, sendo, à partida, seguro na população em estudo (FR B). De facto, os estudos não encontraram diferenças na incidência de descolamento prematuro da placenta, de hemorragia anteparto e pós-parto, nem na incidência de hemorragia neonatal ou anomalias do desenvolvimento. É de salvaguardar, contudo, a possibilidade de vieses importantes na interpretação destes outcomes, sendo necessários mais estudos a longo prazo para avaliar estes e outros potenciais efeitos adversos que, pela sua raridade, necessitam de um follow-up mais alargado.

Uma limitação importante e fonte de heterogeneidade da maior parte dos estudos incluídos nesta revisão reside na disparidade de critérios para definir mulheres grávidas de alto e de baixo risco para desenvolvimento de pré-eclâmpsia, sendo necessários mais estudos para aferir quais os fatores que conferem maior risco para esta doença.

A pré-eclâmpsia é uma das mais graves patologias obstétricas, estando associada a complicações graves e a morbimortalidade materna e fetal, devendo o médico de família estar sensibilizado para o seu rastreio e abordagem. As conclusões deste trabalho podem, assim, melhorar os cuidados às grávidas em risco de pré-eclâmpsia, reduzindo o risco de complicações e melhorando os outcomes maternos e fetais.

No entanto, subsistem ainda algumas dúvidas relativas ao uso de AAS na prevenção da pré-eclâmpsia. Assim sendo, são necessários mais estudos para esclarecer quais os fatores que conferem maior risco para esta patologia, para perceber qual a melhor altura para iniciar a profilaxia com AAS e qual a dose mais adequada. São ainda necessários estudos de boa qualidade para reafirmar a segurança do AAS na população obstétrica.

\section{REFERÊNCIAS BIBLIOGRÁFICAS}

1. Duley L, Henderson-Smart D, Knight M, King J. Antiplatelet drugs for prevention of pre-eclampsia and its consequences: systematic review. BMJ. 2001;322(7282):329-33.

2. World Health Organization. Geographic variation in the incidence of hypertension in pregnancy: World Health Organization international collaborative study of hypertensive disorders of pregnancy. Am J Obstet Gynecol. 1988;158(1):80-3.

3. Khan KS, Wojdyla D, Say L, Gülmezoglu AM, Van Look PF. Who analysis of causes of maternal death: a systematic review. Lancet. 2006;367(9516):1066-74.

4. Tranquilli AL, Dekker G, Magee L, Roberts J, Sibai BM, Steyn W, et al. The classification, diagnosis and management of the hypertensive disor- 
ders of pregnancy: a revised statement from the ISSHP. Pregnancy Hypertens. 2014;4(2):97-104.

5. Mol BW, Roberts CT, Thangaratinam S, Magee LA, de Groot CJ, Hofmeyr GJ. Pre-eclampsia. Lancet. 2016;387(10022):999-1011.

6. Khong TY, De Wolf F, Robertson WB, Brosens I. Inadequate maternal vascular response to placentation in pregnancies complicated by preeclampsia and by small-for-gestational age infants. Br J Obstet Gynaecol. 1986;93(10):1049-59.

7. Schiff E, Peleg E, Goldenberg M, Rosenthal T, Ruppin E, Tamarkin M, et al. The use of aspirin to prevent pregnancy-induced hypertension and lower the ratio of thromboxane $\mathrm{A} 2$ to prostacyclin in relatively high risk pregnancies. N Engl J Med. 1989;321(6):351-6.

8. Roberge $S$, Villa P, Nicolaides $K$, Giguère $Y$, Vainio $M$, Bakthi A, et al. Early administration of low-dose aspirin for the prevention of preterm and term preeclampsia: a systematic review and meta-analysis. Fetal Diagn Ther. 2012;31(3):141-6.

9. Bujold E, Roberge S, Lacasse Y, Bureau M, Audibert F, Marcoux S, et al. Prevention of preeclampsia and intrauterine growth restriction with aspirin started in early pregnancy: a meta-analysis. Obstet Gynecol. 2010;116(2 Pt 1):402-14.

10. Rossi AC, Mullin PM. Prevention of pre-eclampsia with low-dose aspirin or vitamins $C$ and $E$ in women at high or low risk: a systematic review with meta-analysis. Eur J Obstet Gynecol Reprod Biol. 2011;158(1):9-16.

11. Ruano R, Fontes RS, Zugaib M. Prevention of preeclampsia with lowdose aspirin: a systematic review and meta-analysis of the main randomized controlled trials. Clinics (Sao Paulo). 2005;60(5):407-14.

12. Trivedi NA. A meta-analysis of low-dose aspirin for prevention of preeclampsia. J Postgrad Med. 2011;57(2):91-5.

13. Henderson JT, Whitlock EP, O'Connor E, Senger CA, Thompson JH, Rowland MG. Low-dose aspirin for the prevention of morbidity and mortality from preeclampsia: a systematic evidence review for the U.S. Preventive Services Task Force. Ann Intern Med. 2014;160(10):695-703.

14. Ayala DE, Ucieda R, Hermida RC. Chronotherapy with low-dose aspirin for prevention of complications in pregnancy. Chronobiol Int. 2013;30(1-2):260-79.

15. Askie LM, Duley L, Henderson-Smart DJ, Stewart LA. Antiplatelet agents for prevention of pre-eclampsia: a meta-analysis of individual patient data. Lancet. 2007;369(9575):1791-8.

16. Duley L, Henderson-Smart DJ, Meher S, King JF. Antiplatelet agents for preventing pre-eclampsia and its complications. Cochrane Database Syst Rev. 2007;(2):CD004659.

17. Roberge $S$, Giguère $Y$, Villa $P$, Nicolaides $K$, Vainio $M$, Forest JC, et al. Early administration of low-dose aspirin for the prevention of severe and mild preeclampsia: a systematic review and meta-analysis. Am J Perinatol. 2012;29(7):551-6.

18. LeFevre ML. Low-dose aspirin use for the prevention of morbidity and mortality from preeclampsia: U.S. Preventive Services Task Force Recommendation Statement. Ann Intern Med. 2014;161(11):819-26.

19. Magee LA, Pels A, Helewa M, Rey E, von Dadelszen P. Diagnosis, evaluation, and management of the hypertensive disorders of pregnancy. Pregnancy Hypertens. 2014;4(2):105-45.

20. National Collaborating Centre for Women's and Children's Health, National Institute for Health and Clinical Excellence. Hypertension in pregnancy: the management of hypertensive disorders during pregnancy [Internet]. London: Royal College of Obstetricians and Gynaecologists; 2011. Available from: http://www.ncbi.nlm.nih.gov/books/ NBK62649/?report=classic

21. Gillon TE, Pels A, von Dadelszen P, MacDonell K, Magee LA. Hypertensive disorders of pregnancy: a systematic review of international clinical practice guidelines. PLoS One. 2014;9(12):e113715.

\section{CONFLITOS DE INTERESSE}

Os autores declaram não ter conflitos de interesse.

\section{FINANCIAMENTO DO ESTUDO}

Os autores declaram que o trabalho relatado neste manuscrito não foi objeto de qualquer tipo de financiamento externo.

\section{ENDEREÇO PARA CORRESPONDÊNCIA}

Sara Santos Ferreira

USF Infesta, Unidade Local de Saúde de Matosinhos

E-mail: sara_danielapk@hotmail.com

Recebido em 03-02-2016

Aceite para publicação em 22-12-2016 


\section{ABSTRACT}

\section{ASPIRIN IN PREGNANCY FOR THE PREVENTION OF PREECLAMPSIA: AN EVIDENCE-BASED REVIEW}

Objectives: To review the current evidence for the effectiveness of aspirin in preventing preeclampsia.

Data sources: MEDLINE and evidence-based medicine websites.

Methods: We searched for clinical practice guidelines (CPG), randomized controlled trials (RCT), systematic reviews (SR) and meta-analyzes (MA), published between 13/11/2005 and 13/11/2015, using the MESH terms 'pre-eclampsia' and 'aspirin' and the Portuguese DeCS terms 'pre-eclâmpsia' and 'aspirina'. The Strength of Recommendation Taxonomy (SORT) of American Academy of Family Physicians was used for the assignment of levels of evidence (LE) and the strength of recommendations (SOR). Results: A total of 14 articles were selected for this review out of 296 articles found. These included seven MA, three SR, three CPG and one RCT. The SR and MA found benefits for aspirin in the prevention of preeclampsia, particularly in women at highrisk. One SR with LE 2 found no evidence to support the use of aspirin prophylaxis in women at risk for preeclampsia. Studies that analyzed women at low-risk separately found no benefit from the use of aspirin in this group of women. The CPG recommend the use of aspirin in the prevention of preeclampsia in high-risk women and discouraged its use in low-risk women. The RCT supported the same recommendation for high-risk women.

Conclusions: The available evidence suggests that low-dose aspirin is effective for the prevention of preeclampsia in women at risk. There is clear evidence of its benefit in high-risk women (SOR A) and its use is discouraged in low-risk women (SOR A). There is still a need for more high quality studies with similar methods conducted in relevant populations to support these recommendations.

Keywords: Pre-eclampsia; Aspirin. 